\title{
Some Asymptotic Properties of the Transition Densities of One-Dimensional Quasidiffusions
}

By

\author{
Uwe KÜCHLER*
}

\section{§1. Introduction and Preliminary Results}

Let $\left(X_{t}\right)_{t \geqq 0}$ be a quasidiffusion on $[0,1)$ given by its speed measure $m$ and scale $p$. Suppose the boundary point 0 is reflecting regular. If the boundary point 1 is regular we restrict ourselves to the case that $\left(X_{t}\right)$ is killed as soon as it hits 1 , although the arguments hold equally well for all other locally boundary conditions.

As $m$ is not assumed to be strictly increasing besides the classical diffusions birth- and death-processes are also included.

In this introducing chapter we consider semigroups of contractions generated by $\left(X_{t}\right)$ in $L_{2}(m)$ and in a Banach space $B$ of continuous $m$-integrable functions. We construct a spectral expansion of the transition densities of $\left(X_{t}\right)$ making use of M.G. Krein's results on the spectral functions of a string ([10]) and study some of their properties. The results given below generalize known facts for birth- and death-processes ([9]) and for some diffusion processes ([3], [8]). They can be proved by using standard methods of the theory of generalized differential operators ([2],[4],[10]) and therefore some of the proofs are omitted.

In Chapter 2 we will show that the spectra of the infinitesimal operators $D_{m} D_{p}$ of $\left(X_{t}\right)$ in $L_{2}(m)$ and $B$ are identical assuming 1 is not a natural boundary. This is used for studying some boundedness and integrability properties of eigenfunctions of this operator.

The main results of this paper are contained in Chapters 3 and 4. In Chapter 3 we define a transformation of the pair $(m, p)$ using an eigenfunction of $D_{m} D_{p}$. This transformation gives a far reaching duality between accessible

Communicated by K. Itô, March 17, 1978.

* Sektion Mathematik, Technische Universität Dresden, Mommsenstrasse 13, DDR-8027 Dresden, German Democratic Republic. 
and entrance boundaries and elucidates once more the connections between limit circle and limit point cases on the one hand and Feller's boundary classification on the other hand.

Chapter 4 is devoted to the investigation of asymptotic properties of the transition densities $p(t, x, y)$ of $\left(X_{t}\right)$. Assuming 1 is an entrance or an accessible boundary we will show that $p(t, x, y)$ and $\frac{p(t, x, y)}{p(1)-p(y)}$ respectively have strictly positive limits for $y \rightarrow 1$ and/or $t \rightarrow \infty$ in a certain space- and time-weak sense. The asymptotic properties given below are not true in general if 1 is a natural boundary. In this case the spectral properties of $D_{m} D_{p}$ and the asymptotic behaviour of $p(t, x, y)$ are of different type. This is known from the example of Brownian motion.

The results of this paper will be used in [13] to study parabolic functions of quasidiffusions. Let us mention that some asymptotic properties of transition densities of diffusions for a bounded time interval were investigated with somewhat other methods in [19]. In this paper we shall use the theory of the generalized differential operator $D_{m} D_{p}$ and the terminology given in [10] (see also [2]). Some necessary notions and properties are summarized in an appendix.

We denote by $R$ the set of real, by $K$ the set of complex numbers and define $K_{0}:=K \backslash(-\infty, 0]$. A nondecreasing function $m$ from

$$
\begin{aligned}
& J:=[0,1] \text { into }[0, \infty] \text { with } \\
& 0=m(0)<m(x)<m(1-0)=m(1) \quad(x \in(0,1))
\end{aligned}
$$

is called a speed measure, a (strictly) increasing continuous function $p$ from $J$ into $[0, \infty]$ is called a scale, and a pair $(m, p)$ of such functions is said to be a canonical pair. With the same letter $m$ and $p$ we denote the measures generated by $m$ and $p$. We put $I:=J \backslash\{1\}, F:=\operatorname{supp} m$ and $E:=F \backslash\{1\}$.

We will make use of Feller's boundary classification (see e.g. [7], [17]) i.e. with the notation $u(x):=\int_{0}^{x} m d p$ and $v(x):=\int_{0}^{x} p d m(x \in J)$ we shall call the boundary 1 regular if $u(1)<\infty$ and $v(1)<\infty$, entrance if $u(1)=\infty$ and $v(1)<\infty$, pure exit if $u(1)<\infty$ and $v(1)=\infty$ and natural if $u(1)=\infty$ and $v(1)=\infty$. The natural case is divided in pure natural boundary $(p(1)=\infty)$ and inaccessible exit boundary $(p(1)<\infty)$ (shortly: i.a. exit boundary). Regular and pure exit boundaries are called accessible, the other inaccessible. Here the boundary 0 is always regular in an analogous classification. 
Let $(m, p)$ be a canonical pair and $\vartheta$ the set of all complex valued functions $f$ on $I$ such that there exists an $m$-locally integrable function $g=: D_{m} D_{p} f$ and two numbers $a, b \in K$ with

$$
f(x)=a+b p(x)+\int_{0}^{x}(p(x)-p(s)) g(s) m(d s) \quad(x \in I) .
$$

For every $\lambda \in K$ there exist the fundamental solutions

$$
\begin{aligned}
& \varphi(., \lambda) \text { and } \psi(., \lambda) \text { of } D_{m} D_{p} g-\lambda g=0(g \in \vartheta) \text { and } \\
& D_{p}^{-} \varphi(0, \lambda)=\psi(0, \lambda)=0, \varphi(0, \lambda)=D_{p}^{-} \psi(0, \lambda)=1 .
\end{aligned}
$$

It is known that either both fundamental solutions belong to $L_{2}(m)$ for every $\lambda \in K$ or at least one of their linear combinations belongs to $L_{2}(m)$ for every $\lambda \in K_{0}$ depending on $\int_{0}^{1} p^{2} d m<\infty$ or $\int_{0}^{1} p^{2} d m=\infty$ respectively. In the first case we speak of the limit circle case (1cc) and in the second of the limit point case (1pc). In the $1 \mathrm{cc}$ the boundary 1 has to be regular or entrance. If 1 is regular we have the $1 \mathrm{cc}$, if 1 is entrance both $1 \mathrm{cc}$ or $1 \mathrm{pc}$ are possible.

To formulate the first proposition let us define

$$
\begin{aligned}
& \vartheta^{*}:=\left\{f \in \vartheta^{*} \cap L_{2}(m) \mid D_{m} D_{p} f \in L_{2}(m), D_{p}^{-} f(0)=0\right\} \text { and } \\
& \Delta:=\left\{\begin{array}{cc}
\left\{f \in \vartheta^{*} \mid D_{p} f(1)=0\right\} & \text { if } \int_{0}^{1} p^{2} d m<\infty \text { and } 1 \text { is entrance, } \\
\left\{f \in \vartheta^{*} \mid f(1)=0\right\} & \text { if } \int_{0}^{1} p^{2} d m<\infty \text { and } 1 \text { is regular, } \\
\vartheta^{*} & \text { if } \int_{0}^{1} p^{2} d m=\infty .
\end{array}\right.
\end{aligned}
$$

Proposition 1. The restriction $\mathbb{D}_{m} D_{p}$ of $D_{m} D_{p}$ to $\Delta$ is a selfadjoint nonpositive operator in $L_{2}(m)$ and its resolvent $R_{\lambda}$ is given by

$$
R_{\lambda} f(x)=\int_{0}^{1} r_{\lambda}(x, y) f(y) m(d y) \quad\left(f \in L_{2}(m), \lambda \in K_{0}\right)
$$

with

$$
r_{\lambda}(x, y)=r_{\lambda}(y, x)=\varphi(x, \lambda) \chi(y, \lambda) \quad(x, y \in I, x \leqq y)
$$

Here $\chi(., \lambda)$ denotes the unique solution of $D_{m} D_{p} g-\lambda g=0$ belonging to $L_{2}(m)$ and $\left(\right.$ if $\left.\int_{0}^{1} p^{2} d m<\infty\right)$ satisfying $D_{p}^{-} \chi(0, \lambda)=-1$ and $\chi(1, \lambda)=0$ if 1 is regular, $D_{p} \chi(1, \lambda)=0$ if 1 is entrance.

The proof is omitted, see e.g. [1],[2],[4],[15] for similar results. 
Corollary. If $\int_{0}^{1} p^{2} d m=\infty$ and 1 is not natural then all elements of $\Delta$ also satisfy certain boundary conditions. Precisely, for any $f \in \Delta$ it holds

$$
\begin{aligned}
& f(1)=0 \text { if } 1 \text { is a pure exit boundary, } \\
& D_{p} f(1)=0 \text { if } 1 \text { is an entrance boundary. }
\end{aligned}
$$

Proof. The first property follows from $m(1)=\infty$ in the pure exit case. To prove the second we assume 1 is entrance and fix $\lambda>0$. Then every $f \in \Delta$ can be represented by $R_{\lambda} g$ with $g=\lambda f-D_{m} D_{p} f \in L_{2}(m)$. Thus

$$
D_{p} f(x)=\left(D_{p} \chi(x, \lambda)\right) \int_{0}^{x} \varphi(., \lambda) g d m+\left(D_{p} \varphi(x, \lambda)\right) \int_{x}^{1} \chi(., \lambda) g d m .
$$

The boundary 1 is entrance, therefore we have $\lim _{x \uparrow 1} D_{p} \varphi(x, \lambda)<\infty$ (see [2], p. 166) and thus the second integral tends to zero for $x \uparrow 1$. Using (A3) it follows

$$
D_{p} f(1)=\lim _{x \uparrow 1}(\varphi(x, \lambda))^{-1} \int_{0}^{x} \varphi(., \lambda) g d m .
$$

(The limit exists and is finite because $\varphi(., \lambda)$ is increasing and $g \in L_{2}(m) \subset L_{1}(m)$ if 1 is entrance.) Thus we have

$$
f(x) \sim p(x) D_{p} f(1) \text { for } x \uparrow 1 .
$$

Now $\int_{0}^{1} p^{2} d m=\infty$ and $f \in L_{2}(m)$ imply $D_{p} f(1)=0$.

Q.E.D.

Let $(m, p)$ be a canonical pair and $\tau$ its main spectral function (see the appendix). We define

$$
p(t, x, y):=\int_{-\infty}^{0} e^{\lambda t} \varphi(x, \lambda) \varphi(y, \lambda) \tau(d \lambda) \quad(t \in K, \operatorname{Re} t>0 ; x, y \in I) .
$$

By virtue of (A1-2) these integrals converge uniformly in $x, y \in[0, c]$ and $t \geqq t_{0}$ for every $c<1$ and $t_{0}>0$ (uniformly in $x, y \in[0,1]$ and $t \geqq t_{0}$ for every $t_{0}>0$ if 1 is regular). Without proof we mention that $t \rightarrow p(t, x, y)$ is holomorphic in $t$ with $\operatorname{Re} t>0$ for every $x, y \in I(x, y \in J$ if 1 is regular).

\section{Proposition 2. The formula}

$$
\text { (4) } \quad T_{t} f(x):=\int_{0}^{1} p(t, x, y) f(y) m(d y) \quad\left(f \in L_{2}(m), x \in I, t \in K, \operatorname{Re} t>0\right)
$$

defines a holomorphic semigroup $\left\{T_{t} \mid t \in K, \operatorname{Re} t>0\right\}$ of selfadjoint contractions $T_{t}$ on $L_{2}(m)$ being strongly continuous at $t=0$ with $T_{0}:=I$ and having $\mathbb{D}_{m} \mathbb{D}_{p}$ as its infinitesimal operator. Furthermore we have 
(5)

$$
p(t, ., y) \in \Delta, \mathbb{D}_{m} \mathbb{D}_{p} p(t, x, y)=\frac{\partial}{\partial t} p(t, x, y) \quad(t \in K, \operatorname{Re} t>0 ; x, y \in I)
$$

and

(6)

$$
p(t, x, y)=p(t, y, x)>0 \quad(t>0, x, y \in I) .
$$

Proof. It is known (see e.g. [2], [10]) that the generalized Fourier transformation $U$ from $L_{2}(m)$ given by

$$
(U f)(\lambda)=\int_{0}^{1} f(x) \varphi(x, \lambda) m(d x) \quad\left(f \in L_{2}(m)\right)
$$

maps $L_{2}(m)$ isometrically onto $L_{2}(\tau)$ and that for the inverse mapping $U^{-1}$ holds

$$
\left(U^{-1} F\right)(x)=\int_{-\infty}^{0} F(\lambda) \varphi(x, \lambda) d \tau(\lambda) \quad\left(F \in L_{2}(\tau)\right) .
$$

The mapping $Q:=U \mathbb{D}_{m} \mathbb{D}_{p} U^{-1}$ is the operator of multiplication acting in $L_{2}(\tau):$

$$
(Q F)(\lambda)=\lambda F(\lambda) \quad\left(F \in \tilde{\Delta}:=\left\{F \in L_{2}(\tau) \mid Q F \in L_{2}(\tau)\right\}\right) .
$$

By standard methods it can be shown that with the definition $T_{t}:=U^{-1} e^{Q t} U$ $(t \in K, \operatorname{Re} t>0)$ the first part of the proposition holds.

From (A1-2) it follows that for every $y \in I$ and $t$ with $\operatorname{Re} t>0$ the function $\lambda \rightarrow e^{\lambda t} \varphi(y, \lambda)$ belongs to $\widetilde{\Delta}$. Therefore $p(t, x, y)=U^{-1}\left(e^{\cdot t} \varphi(y,).\right)(x)$ as a function of $x$ belongs to $\Delta$ and we get

$$
\mathbb{D}_{m} \mathbb{D}_{p} p(t, x, y)=U^{-1}\left(Q e^{s t} \varphi(y, .)\right)(x)=\int_{-\infty}^{0} \lambda e^{\lambda t} \varphi(x, \lambda) \varphi(y, \lambda) \tau(d \lambda) .
$$

Thus from the uniform convergence of the last integral in $(t, x, y)$ with $\operatorname{Re} t \geqq t_{0}$; $x, y \in[0, c]$ for every $t_{0}>0, c<1$ we obtain that this integral is equal to $\frac{\partial}{\partial t} p(t, x, y)$. Hence (5) is proved.

Now we shall show (6). The symmetry of $p(t, x, y)$ is trivial by definition. Let $\lambda>0$. If $f \in L_{2}(m), f \geqq 0$ then $R_{\lambda} f \geqq 0$ by the positiveness of the kernel $r_{\lambda}(x, y)$. The Hille-Yosida-theorem (see e.g. [18]) implies that $T_{t} f \geqq 0$ for all $f \in L_{2}(m)$ with $f \geqq 0$ and every $t>0$. Using the continuity of $p(t, x, y)$ it is easy to see that $p(t, x, y) \geqq 0(t>0, x, y \in I)$. Suppose $p\left(t_{0}, x_{0}, y_{0}\right)=0$ for a certain tripel $\left(t_{0}, x_{0}, y_{0}\right)$ with $t_{0}>0 ; x_{0}, y_{0} \in I$. We can assume $x_{0}, y_{0}$ are points of increasing of $m$ (because $p(t, x,$.$) and p(t, ., y)$ depend linear in scale $p$ on intervals where $m$ is constant).

Now from the semigroup property of $\left(T_{t}\right)$ we have for every $h \in\left(0, t_{0}\right)$ 


$$
\int_{0}^{1} p\left(t_{0}-h, x_{0}, z\right) p\left(h, z, y_{0}\right) m(d z)=p\left(t_{0}, x_{0}, y_{0}\right)=0 .
$$

Let $h^{\prime} \in\left(0, t_{0}\right)$ be fixed. Using $T_{h^{\prime}} \neq 0$ it follows $p\left(h^{\prime}, z^{\prime}, y_{0}\right)>0$ for some $z^{\prime}$ $\in I$. By continuity we have $p\left(h, z, y_{0}\right)>0$ in some neighbourhood of $\left(h^{\prime}, z^{\prime}\right)$. Hence (7) implies $p\left(t_{0}-h, x_{0}, z\right)=0$ for all $(h, z)$ in some neighbourhood of $\left(h^{\prime}, z^{\prime}\right)$. Thus by the holomorphy of $p\left(., x_{0}, z^{\prime}\right)$ it follows that $p\left(s, x_{0}, z^{\prime}\right)=0$ for every $s>0$. Therefore $\int_{0}^{\infty} e^{-\lambda s} p\left(s, x_{0}, z^{\prime}\right) d s=0(\lambda>0)$. But this integral is equal to $r_{\lambda}\left(x_{0}, z^{\prime}\right)$, because $x_{0}$ is an increasing point of $m$ (see (A6)). This contradicts $r_{\lambda}(x, y)>0$ $(x, y \in I)$. Hence (6) holds.

Q.E.D.

Remark. Without proof let us mention that $P_{t}(x, A):=\int_{A} p(t, x, y) m(d y)$ are the transition probabilities of a strongly Fellerian stochastically continuous Markov process with state space $E$. This process is reflected at the boundary 0 and killed as soon as it hits the boundary 1 . It is called the quasidiffusion corresponding to $(m, p)$ and the boundary conditions mentioned above (see e.g. [7], [16], [20]), or, because the boundary conditions are fixed here, shortly corresponding to $(m, p)$. In this sense we call $p(t, x, y)$ the transition densities corresponding to $(m, p)$.

To obtain further informations about the function $p(t, x, y)$ we shall study corresponding semigroups in spaces of continuous and $m$-integrable functions. To this purpose we take into consideration the Banach space $(C,\|\|$.$) of$ continuous functions from $J$ into $K$ being linear in scale $p$ on intervals where $m$ is constant. The norm $\|$. $\|$ is given by $\|f\|:=\sup _{x \in J}\|f(x)\|(f \in C)$. Moreover let $C_{0}\left(C_{1}\right)$ be the subset of all $f \in C$ such that $f(1)=0 \quad\left(\int_{0}^{1}|f| d m<\infty\right.$ resp.) Then $\left(C_{0},\|\|.\right)$ and $\left(C_{1}, \|\right.$. $\left.\|_{1}\right)$ with $\|f\|_{1}:=\max \left(\|f\|, \int_{0}^{1}|f| d m\right)\left(f \in C_{1}\right)$ are also Banach spaces.

We will study the restrictions of $D_{m} D_{p}$ to $C_{1} C_{0}$ and $C_{1}$ and we will show that some eigenfunctions of these restrictions are $m$-integrable. This is obvious if 1 is entrance or regular, because in this case $m(1)<\infty$ (i.e. $C \subset L_{1}(m)$ ) holds. If 1 is pure exit we have $m(1)=\infty$ and thus we must apply another methods to prove the $m$-integrability of the eigenfunctions. Let us define

$$
(B,|\cdot|)=\left\{\begin{array}{l}
(C,\|\cdot\|) \text { if } 1 \text { is inaccessible, } \\
\left(C_{0},\|\cdot\|\right) \text { if } 1 \text { is regular, } \\
\left(C_{1},\|\cdot\|_{1}\right) \text { if } 1 \text { is pure exit. }
\end{array}\right.
$$


For every $\lambda>0$ we define by

$$
R_{\lambda} f(x):=\int_{0}^{1} r_{\lambda}(x, y) f(y) m(d y)
$$

a linear mapping $R_{\lambda}$ on the set of functions $f$ such that the integral in (8) exists.

Proposition 3. The formula

$$
S_{t} f(x):=\int_{0}^{1} p(t, x, y) f(y) m(d y), S_{0} f:=f(f \in B, t>0)
$$

defines a strongly continuous semigroup $\left\{S_{t} \mid t \geqq 0\right\}$ of contractions $S_{t}$ on $(B, \mid$. |). The infinitesimal operator of $\left(S_{t}\right)$ is the restriction of $D_{m} D_{p}$ to $\Delta_{B}:\{f \in \vartheta \cap$ $\left.B \mid D_{m} D_{p} f \in B\right\}$, and for $\lambda>0$ the corresponding resolvent operator $R_{\lambda}$ on $B$ is given by (8).

The proof of this proposition can also be given by standard methods and is omitted here.

\section{$\S 2$. Spectral Properties of $D_{m} D_{p}$}

In the following theorem we consider the spectra of the restrictions of $D_{m} D_{p}$ to $\Delta \subset L_{2}(m)$ and to $\Delta_{B} \subset B$ which are denoted by $\sigma$ and $\sigma_{B}$ respectively.

Theorem 1. Let 1 be an accessible or an entrance boundary. Then the spectra $\sigma$ and $\sigma_{B}$ coincide and consist of a strictly decreasing sequence $\left(\lambda_{n}\right.$, $n \geqq 0$ ) of nonpositive simple eigenvalues $\lambda_{n}(n \geqq 0)$ having no finite accumulation points. The eigenfuntion corresponding to $\lambda_{n}$ is $\varphi\left(., \lambda_{n}\right)$. We have

$$
\begin{aligned}
& \varphi\left(x, \lambda_{0}\right)>0(x \in I) \text { and } \\
& \lambda_{0}<0 \text { if } 1 \text { is accessible, } \\
& \lambda_{0}=0 \text { if } 1 \text { is entrance. }
\end{aligned}
$$

Proof. Let $\lambda>0$. It is easy to see that $R_{\lambda}$ on $L_{2}(m)$ is a compact operator. Indeed under the assumptions of the theorem we have

$$
\int_{0}^{1} r_{\lambda}(x, x) m(d x)<\infty .
$$

This follows from $r_{\lambda}(x, x) \leqq \chi(0, \lambda) \varphi(x, \lambda)$ and $D_{p} \varphi(1, \lambda)=\lambda \int_{0}^{1} \varphi(x, \lambda) m(d x)<\infty$ if 1 is regular or entrance, $r_{\lambda}(x, x) \leqq \varphi(1, \lambda) \chi(x, \lambda)$ and $\varphi(1, \lambda) \int_{0}^{1} \chi(x, \lambda) m(d x)<\infty$ if 1 is pure exit (see (A4) and [2], p. 166). Remarking $r_{\lambda}^{2}(x, y) \leqq r_{\lambda}(x, x) r_{\lambda}(y, y)$, (11) implies $\iint r_{\lambda}^{2} d m d m<\infty$, i.e. $R_{\lambda}$ is compact in $L_{2}(m)$. 
The properties of its spectrum imply that $\sigma$ consists of a sequence $\left(\lambda_{n}\right.$, $n \geqq 0$ ) of eigenvalues having no finite accumulation point. The corresponding eigenfunctions $g_{n}$ satisfy the condition $D_{p}^{-} g_{n}(0)=0$. Thus $g_{n}=\varphi\left(., \lambda_{n}\right)$ which proves the simplicity of $\lambda_{n}$. From the nonpositivity of $\boldsymbol{D}_{m} \boldsymbol{D}_{p}$ it follows $\lambda_{n} \leqq 0$. Let $\left(\lambda_{n}\right)$ be ordered: $0 \geqq \lambda_{0}>\lambda_{1}>\cdots>\lambda_{n}>\cdots$. To continue the proof we shall prove the following

Lemma 1. For every $\lambda>0$ the operator $R_{\lambda}$ is compact on $(B,|\cdot|)$.

Proof. If 1 is regular, then $r_{\lambda}(.,$.$) is continuous on [0,1]^{2}$, and from $m(1)<$ $\infty$ as usual (see e.g. [8]) it follows the compactness of $R_{\lambda}$ in $(C,\|\|$.$) and$ therefore in $\left(C_{0},\|\|.\right)$. If 1 is entrance we have from $m(1)<\infty$ and from the continuity of $r_{\lambda}(.,$.$) on [0,1)^{2}$ that for every $n$ the operator $R_{\lambda}^{(n)}$ generated by $r_{\lambda}^{(n)}(.,)=.\min \left(r_{\lambda}(.,), n.\right)$ is compact in $(C,\|\|$.$) . By the Lagrange identity it$ follows $D_{p}(\varphi(x, \lambda) \chi(x, \lambda))=2 \chi(x, \lambda) D_{p} \varphi(x, \lambda)-1$. Because 1 is entrance we have $\chi(1, \lambda)>0$ and $D_{p} \varphi(1, \lambda)<\infty$ (see [2], p.166). Furthermore $\chi(1, \lambda) D_{p} \varphi(1, \lambda)=1$ holds (see (A3)). Thus $\lim _{x \uparrow 1} D_{p}(\varphi(x, \lambda) \chi(x, \lambda))=1$. Consequently the function $\varphi(., \lambda) \chi(., \lambda)$ increases near 1 and converges to $\infty$ for $x \uparrow 1$. Thus there exists a sequence $\left(x_{n}\right) \uparrow 1$ such that $\varphi\left(x_{n}, \lambda\right) \chi\left(x_{n}, \lambda\right)=n$. Hence $r_{\lambda}^{(n)}(x, y)=r_{\lambda}(x, y)$ if $x$ or $y \leqq x_{n}$. Using $D_{p} \varphi(1, \lambda)<\infty$ it follows

$$
\begin{aligned}
&\left\|R_{\lambda}^{(n)}-R_{\lambda}\right\|=\sup _{x} \int_{0}^{1}\left(r_{\lambda}(x, y)-r_{\lambda}^{(0)}(x, y)\right) m(d y) \\
& \leqq \sup _{x \geqq x_{n}}\left(\chi(x, \lambda) \int_{x_{n}}^{x}\left(\varphi(y, \lambda)-\varphi\left(x_{n}, \lambda\right)\right) m(d y)+\varphi(x, \lambda) \int_{x}^{1}(\chi(y, \lambda)-\chi(1, \lambda)) m(d y)\right) \\
& \leqq \frac{\chi(0, \lambda)}{\lambda}\left(D_{p} \varphi(1, \lambda)-D_{p} \varphi\left(x_{n}, \lambda\right)\right)+\frac{(\chi(0, \lambda)-\chi(1, \lambda))}{\lambda}\left(D_{p} \varphi(1, \lambda)-D_{p} \varphi\left(x_{n}, \lambda\right)\right) \\
& \underset{n \rightarrow \infty}{\longrightarrow} 0 .
\end{aligned}
$$

Therefore $R_{\lambda}$ is compact in $(C,\|\|$.$) if 1$ is entrance.

Let 1 be pure exit. At first we will show that for $S:=\{f \in C \mid\|f\| \leqq 1\}$ the set $R_{\lambda} S$ is totally bounded. This implies that $R_{\lambda}$ is compact on $(C, \|$. $\|)$. Of course $R_{\lambda} S$ is a bounded set. Using (A3) we have

$$
\begin{aligned}
& \left(R_{\lambda} 1\right)(x)=\chi(x, \lambda) \int_{0}^{x} \varphi(., \lambda) d m+\varphi(x, \lambda) \int_{x}^{1} \chi(., \lambda) d m \\
= & \frac{1}{\lambda}\left(\chi(x, \lambda) D_{p} \varphi(x, \lambda)+\varphi(x, \lambda)\left(D_{p} \chi(1, \lambda)-D_{p} \chi(x, \lambda)\right)\right)
\end{aligned}
$$




$$
=\frac{1}{\lambda}\left(1+\varphi(x, \lambda) D_{p} \chi(1, \lambda)\right)=\frac{1}{\lambda}\left(1-\frac{\varphi(x, \lambda)}{\varphi(1, \lambda)}\right) .
$$

Thus for every $\varepsilon>0$ there exists a $\delta=\delta(\varepsilon)$ such that $\left(R_{\lambda} 1\right)(x)<\varepsilon$ for every $x$ with $x \geqq 1-\delta$. Therefore $\left|R_{\lambda} f(x)\right|<\varepsilon$ for every $f \in S$ and every $x \geqq 1-\delta$. Now it is easy to show that for every $\varepsilon>0$ there exists a finite set of points $x_{i}$ with $0<x_{1} \leqq \cdots \leqq x_{n}<1-\delta(\varepsilon)$ such that $\left|R_{\lambda} f(x)-R_{\lambda} f\left(x_{i}\right)\right|<\varepsilon$ for every $x \in\left[x_{i-1}, x_{i+1}\right](i=1, \cdots, n-1)$ with $x_{0}=0$ and $x_{n+1}=1-\delta(\varepsilon)$. Thus the total boundedness of $R_{\lambda}$ is proved.

Now we have to show that $R_{\lambda}$ is compact in $\left(C_{1},\|.\|_{1}\right)$ if 1 is pure exit. Let $\left(f_{n}\right)$ be a sequence in $C_{1}$ with $\left\|f_{n}\right\|_{1} \leqq 1$. Then by the compactness of $R_{\lambda}$ in $C$ there exists a subsequence $\left(f_{n^{\prime}}{ }^{\prime}\right)$ converging with respect to the norm of $C$. The elements of $C_{1}$ can be identified with elements of the dual $C^{*}: f \rightarrow F_{f}(g):=$ $\int_{0}^{1} f \cdot g d m\left(f \in C_{1}, g \in C\right)$.

The dual operator $R_{\lambda}^{*}$ of $R_{\lambda}$ in $C^{*}$ is also compact and from the symmetry of $r_{\lambda}\left(.\right.$, . .) it follows that $R_{\lambda}^{*} F_{f}$ can be identified with $R_{\lambda} f$ if $f \in C_{1}$. Hence there exists a subsequence $\left(f_{n^{\prime \prime}}\right)$ of $\left(f_{n^{\prime}}\right)$ converging in the sense of $L_{1}$-norm and thus in the sense of the norm $\|$. $\|_{1}$. Summarizing we have proved that $R_{\lambda}$ for $\lambda>0$ is a compact operator in $(B,|| \mid)$. In particular the spectrum $\sigma_{B}$ is a sequence of eigenvalues having no finite accumulation point.

To continue the proof of Theorem 1 we have to show that $\sigma_{B}$ and $\sigma$ are identical. We remark that $B \subset L_{2}(m)$ and that $B$ is dense in $L_{2}(m)$. Moreover there exists a constant $C>0$ such that

$$
\|f\|_{L_{2}} \leqq C\|f\| \quad(f \in B)
$$

Obviously every eigenvalue of $D_{m} D_{p}$ in $B$ is an eigenvalue of $\mathbb{D}_{m} D_{p}$ in $L_{2}(m)$, thus $\sigma_{B} \subseteq \sigma$. If $\mu \in R_{1}$ not belongs to $\sigma_{B}$ then $R_{\mu}$ is a bounded linear operator in $B$ which has by (12) and by the symmetry property of $r_{\lambda}(x, y)$ a bounded extension $R_{\mu}^{\prime}$ to $L_{2}(m)$ (see [8], [12]). Using the closeness of $\mathbb{D}_{m} \mathbb{D}_{p}$ it is easy to show that $R_{\mu}^{\prime}$ is the inverse of $\left(\mu I-D_{m} D_{p}\right)$ i.e. $\mu \notin \sigma$ and therefore it holds $\sigma \subseteq \sigma_{B}$.

Now we shall show the positiveness of $\varphi\left(., \lambda_{0}\right)$. If $\lambda_{0}=0$ then $\varphi\left(., \lambda_{0}\right)=1>0$. Let $\lambda_{0}<0$ and $\lambda>0$. Then $\frac{1}{\lambda-\lambda_{0}}>0$ is the greatest eigenvalue of the compact and positive linear operator $R_{\lambda}$ in the Banach lattice $(B,\|\cdot\|$. Thus the eigenfunction $\varphi\left(., \lambda_{0}\right)$ is nonnegative. Suppose $x_{0}<1$ is the first zero of $\varphi\left(., \lambda_{0}\right)$. 
Then $D_{p} \varphi\left(x_{0}, \lambda_{0}\right)=\lambda_{0} \int_{0}^{x_{0}} \varphi\left(s, \lambda_{0}\right) m(d s)<0$ and thus $\varphi\left(x, \lambda_{0}\right)=\int_{x_{0}}^{x} D_{p} \varphi\left(s, \lambda_{0}\right) p(d s)<0$ for some $x$ in neighbourhood of $x_{0}$. This contradicts $\varphi\left(., \lambda_{0}\right) \geqq 0$. Thus $\varphi\left(x, \lambda_{0}\right)>0$ if $x \in I$. If 1 is accessible we have $\int_{-\infty}^{0} \frac{\tau(d \lambda)}{|\lambda|}=\sum_{0}^{\infty} \frac{\tau_{k}}{\left|\lambda_{k}\right|}=\Gamma(0)=p(1)<\infty$. Thus $\lambda_{0} \neq 0$, i.e. $\lambda_{0}<0$. (See the appendix for notations.) If 1 is entrance we have $\varphi(., 0) \equiv 1 \in \Delta_{B}$ and therefore $\lambda_{0}=0$.

Q.E.D.

Corollary. We have

$$
\begin{aligned}
& \varphi\left(1, \lambda_{n}\right)=0,\left|D_{p} \varphi\left(1, \lambda_{n}\right)\right|<\infty \text { if } 1 \text { is accessible and } \\
& \left|\varphi\left(1, \lambda_{n}\right)\right|<\infty, D_{p} \varphi\left(1, \lambda_{n}\right)=0 \text { if } 1 \text { is entrance. }
\end{aligned}
$$

Proof. Theorem 1 implies $\varphi\left(., \lambda_{n}\right) \in L_{1}(m)$ if 1 is accessible or entrance. Therefore $\left|D_{p} \varphi\left(1, \lambda_{n}\right)\right|=\left|\lambda_{n} \int_{0}^{1} \varphi\left(x, \lambda_{n}\right) m(d x)\right|<\infty$ in both cases. The other properties follow from $\varphi\left(., \lambda_{n}\right) \in \Delta \cap \Delta_{B}$ and the corollary after Proposition 1.

U.E.D.

Let 1 be accessible or entrance and $\left(\lambda_{k}\right)$ the sequence of eigenvalues of $\boldsymbol{D}_{\boldsymbol{m}} \boldsymbol{D}_{\boldsymbol{p}}$. We know from (A1-2) that the series

$$
p(t, x, y)=\sum_{0}^{\infty} e^{\lambda_{k} t} \varphi\left(x, \lambda_{k}\right) \varphi\left(y, \lambda_{k}\right) \tau_{k}
$$

converge uniformly in $(t, x, y)$ with $t \geqq t_{0} ; x, y \leqq c$ for every $t_{0}>0$ and $c<1$. If 1 is accessible somewhat more holds. (See also the remark (ii) below.)

Proposition 4. Assume 1 is accessible. Then the series (13) converge uniformly in $t \geqq t_{0}$ and $x, y<1$ for every $t_{0}>0$.

Proof. Let $\lambda>0$. Then by $r_{\lambda}(x,.) \in L_{2}(m)$ for every $x \in I$ it follows

$$
r_{\lambda}(x, .)=\sum_{k=0}^{\infty} \frac{\varphi\left(x, \lambda_{k}\right) \varphi\left(., \lambda_{k}\right)}{\lambda-\lambda_{k}} \tau_{k} \quad(x \in I)
$$

in the sense of $L_{2}(m)$-convergence. Thus for the operator $R_{\lambda}^{(n)}$ generated by the kernel

$$
r_{\lambda}(x, y, n)=r_{\lambda}(x, y)-\sum_{k=0}^{n} \frac{\varphi\left(x, \lambda_{k}\right) \varphi\left(y, \lambda_{k}\right)}{\lambda-\lambda_{k}} \tau_{k} \quad(x, y \in I)
$$

we have

$$
\left(R_{\lambda}^{(n)} f, f\right) \geqq 0 \quad\left(f \in L_{2}(m)\right)
$$


Therefore $r_{\lambda}(x, x, n) \geqq 0$ for every $x \in E$, i.e.

$$
r_{\lambda}(x, x) \geqq \sum_{k=0}^{n} \frac{\left(\varphi\left(x, \lambda_{k}\right)\right)^{2}}{\lambda-\lambda_{k}} \tau_{k} \quad(x \in E) .
$$

The accessibility of 1 implies that $r_{\lambda}(x, x)$ is bounded in $x$. Therefore the series on the right side converge uniformly in $x \in E$ and thus also in $x<1$. From

$$
\sum_{n}^{m}\left|\frac{\varphi\left(x, \lambda_{k}\right) \varphi\left(y, \lambda_{k}\right)}{\lambda-\lambda_{k}}\right| \tau_{k} \leqq\left\{\sum_{n}^{m} \frac{\left|\varphi\left(x, \lambda_{k}\right)\right|^{2}}{\lambda-\lambda_{k}} \tau_{k} \sum_{n}^{m} \frac{\left|\varphi\left(y, \lambda_{k}\right)\right|^{2}}{\lambda-\lambda_{k}} \tau_{k}\right\}^{1 / 2}
$$

and $e^{\lambda_{k} t_{0}} \leqq \frac{1}{\lambda-\lambda_{k}}$ for sufficiently large $k$ the proposition follows.

Q.E.D.

By the same conclusions as in the preceding proof it can be shown that (14) holds also if 1 is entrance. Thus from (11) it follows

Corollary. If 1 is accessible or entrance then

$$
\sum_{1}^{\infty} \frac{1}{\left|\lambda_{k}\right|}<\infty
$$

Remarks. (i) It can be shown that the $(k+1)$-th eigenfunction $\varphi\left(., \lambda_{k}\right)$ has exactly $k$ zeros in $(0,1)$ if 1 is accessible or entrance. (See e.g. [3] for the method).

(ii) Let 1 be entrance and $\int_{0}^{1} p^{2} d m<\infty$. Then the series (13) also converge uniformly in $x, y<1$ and $t \geqq t_{0}$ for every $t_{0}>0$. The proof is included in the proof of Theorem 3 below.

\section{§3. A Transformation of the Canonical Pair}

In this chapter we will show that there exists a far reaching connection between accessible and entrance boundaries. By a relatively simple transformation of $(m, p)$ in a new canonical pair $\left(m^{*}, p^{*}\right)$ the type of the boundary 1 changes, and under this transformation regular and entrance boundaries being in $1 \mathrm{cc}$, pure exit and entrance boundaries being in $1 \mathrm{pc}$ correspond to another (see Propositions 5 and 5' below).

Let $(m, p)$ be a canonical pair, $\tau$ the corresponding main spectral function and $\lambda_{0}:=\sup \operatorname{supp} \tau$. We recall $\lambda_{0} \leqq 0$.For every $\mu \geqq \lambda_{0}$ we introduce a new canonical pair $\left(m^{(\mu)}, p^{(\mu)}\right)$ by

$$
d m^{(\mu)}:=\varphi^{2}(., \mu) d m, \quad d p^{(\mu)}:=\varphi^{-2}(., \mu) d p .
$$


Obviously $d m^{(0)}=d m$ and $d p^{(0)}=d p$.

Let $\varphi^{(\mu)}, \psi^{(\mu)}$ and $\tau^{(\mu)}$ be the fundamental solutions and the main spectral function of $\left(m^{(\mu)}, p^{(\mu)}\right)$ respectively. (More generally, all symbols connected with $\left(m^{(\mu)}, p^{(\mu)}\right)$ get the superscript ${ }^{(\mu)}$.)

Lemma 2. The following properties hold:

(i) $\quad \varphi^{(\mu)}(x, \lambda)=\frac{\varphi(x, \lambda+\mu)}{\varphi(x, \mu)}, \psi^{(\mu)}(x, \lambda)=\frac{\varphi(x, \lambda+\mu)}{\varphi(x, \mu)} \quad(x \in I, \lambda \in K)$

(ii) $\tau^{(\mu)}(\lambda)=\tau(\lambda+\mu) \quad(\lambda \in(-\infty, \infty))$

(iii) $\left(\left(m^{(\mu)}\right)^{(\eta)},\left(p^{(\mu)}\right)^{(\eta)}\right)=\left(m^{(\mu+\eta)}, p^{(\mu+\eta)}\right) \quad\left(\mu, \mu+\eta \geqq \lambda_{0}\right)$.

Proof. Using [10], p. 661, (2.23) and an analogous formula for $\varphi(., \mu)$ and $\psi(., \lambda+\mu)$ it follows (i). From $\Gamma^{(\mu)}(\lambda)=\lim _{x \uparrow_{1}} \frac{\psi^{(\mu)}(x, \lambda)}{\varphi^{(\mu)}(x, \lambda)}=\Gamma(\lambda+\mu)$ we have (ii). (iii) can be shown by an easy calculation.

Q.E.D.

Now we study the character of the boundary point 1 with respect to $\left(m^{(\mu)}\right.$, $\left.p^{(\mu)}\right)$. To this purpose we define

$$
\begin{array}{ll}
u^{(\mu)}(x):=\int_{0}^{x} m^{(\mu)} d p^{(\mu)}=\int_{0}^{x} \int_{0}^{s} \frac{\psi^{2}(t, \mu)}{\varphi^{2}(s, \mu)} d m d p & \text { and } \\
v^{(\mu)}(x):=\int_{0}^{x} p^{(\mu)} d m^{(\mu)}=\int_{0}^{x} \psi(s, \mu) \varphi(s, \mu) d m & (x \in J) .
\end{array}
$$

The following lemma is useful for studying the given transformation but is not needed in the sequel explicitely. Therefore we shall omit the proof.

Lemma 3. If $p(1)<\infty, \mu>\lambda_{0}$ or $p(1)=\infty, \mu=\lambda_{0}$ then the character of the boundary 1 with respect to $\left(m^{(\mu)}, p^{(\mu)}\right)$ is the same as to $(m, p)$.

Lemma 3 shows that a change of the character of 1 may be only if $p(1)<\infty$ and $\mu=\lambda_{0}$ or if $p(1)=\infty$ and $\mu>\lambda_{0}$.

In the first case the boundary 1 is an accessible or an i.a. exit boundary with respect to $(m, p)$, in the second it is an entrance or a natural boundary with respect to $(m, p)$.

Proposition 5. Let $p(1)<\infty$ and $\mu=\lambda_{0}$. Then the boundary 1 is

(i) $\left(m^{(\mu)}, p^{(\mu)}\right)$-entrance (lcc) if 1 is $(m, p)$-regular,

(ii) $\left(m^{(\mu)}, p^{(\mu)}\right)$-entrance $(l p c)$ if 1 is $(m, p)$-pure exit,

(iii) $\left(m^{(\mu)}, p^{(\mu)}\right)$-natural, $\quad$ if 1 is $(m, p)$-i.a. exit.

Proof. Suppose $p(1)<\infty, \mu=\lambda_{0}$ and let be at first $u(1)<\infty$. Thus $\lambda_{0}$ is 
a point of discontinuity of $\tau$, and therefore

$$
p^{(\mu)}(1)=\Gamma(\mu)=\Gamma\left(\lambda_{0}\right)=\int_{-\infty}^{0} \frac{d \tau(\lambda)}{\lambda_{0}-\lambda}=\infty \text { holds. }
$$

(i): If 1 is $(m, p)$-regular we have $\int_{0}^{1} p^{2} d m<\infty$. This implies

$$
\psi\left(., \lambda_{0}\right) \in L_{2}(m), \text { i.e. } \int_{0}^{1}\left(p^{(\mu)}\right)^{2} d m^{(\mu)}=\int_{0}^{1}(\psi(., \mu))^{2} d m<\infty .
$$

Therefore 1 is $\left(m^{(\mu)}, p^{(\mu)}\right)$-nonregular with $1 c c$, this means $\left(m^{(\mu)}, p^{(\mu)}\right)$-entrance with 1cc. Thus (i) is proved.

(ii): If 1 is $(m, p)$-pure exit then $\mu=\lambda_{0}<0$ and thus $0<\psi(x, \mu)<p(x)<\infty$ for all $x \leqq 1$. It follows

$$
v^{(\mu)}(1)=\int_{0}^{1} \psi(x, \mu) \varphi(x, \mu) m(d x) \leqq \psi(1, \mu) D_{p} \varphi(1, \mu) \frac{1}{\mu}<\infty .
$$

From $p^{(\mu)}(1)=\infty$ we have $u^{(\mu)}(1)=\infty$. Thus 1 has to be $\left(m^{(\mu)}, p^{(\mu)}\right)$ entrance. Using the inequality

$$
\int_{0}^{1}\left(p^{(\mu)}\right)^{2} d m^{(\mu)}=\int_{0}^{1} \psi^{2}(., \mu) d m \geqq \psi^{2}(1, \mu) m(1)=\infty,
$$

it follows that in this case 1 is in the $\left(m^{(\mu)}, p^{(\mu)}\right)-1$ pc. Therefore (ii) is proved.

(iii): Let 1 be an $(m, p)$-i.a. exit (in particular $u(1)=\infty)$. Let us assume $\mu=\lambda_{0}<0$ (otherwise we have $\lambda_{0}=0$ and the conclusion is obvious). We have

$$
\begin{aligned}
& \int_{0}^{1} r_{-\mu}^{(\mu)}(x, x) m^{(\mu)}(d x)=\int_{0}^{1} \varphi\left(x, \lambda_{0}\right) \chi\left(x, \lambda_{0}\right) m(d x)=\int_{0}^{1}(p(1)-p(x)) m(d x) \\
= & u(1)=\infty .
\end{aligned}
$$

Thus 1 cannot be $\left(m^{(\mu)}, p^{(\mu)}\right)$-accessible or -entrance because in this cases the trace of $R_{\lambda}^{(\mu)}$ is finite ((see (16)). Therefore (iii) holds. Q.E.D.

Remark. In (iii) of Pproposition 5 the boundary 1 need not be $\left(m^{(\mu)}, p^{(\mu)}\right)$ pure natural. For example let $(m, p)$ be the canonical pair corresponding to the spectral function $\tau(\mu)=-\min \left(\mu^{2}, 1\right)(\mu \leqq 0)$, which exists by the solution of the inverse spectral problem (see [10]). Then $\lambda_{0}=0, p(1)=\int_{-\infty}^{0} \frac{d \tau}{\mu}=2<\infty$. Therefore 1 is an $(m, p)$ - and $\left(m^{(\mu)}, p^{(\mu)}\right)$-i.a. exit.

The following proposition is the converse of Proposition 5 in a certain sense. We remark that $p(1)=\infty$ implies that 1 is $(m, p)$-entrance or -pure natural. 
Proposition $5^{\prime}$. Let $p(1)=\infty$ and $\mu>\lambda_{0}$. Then the boundary 1 is

(i') $\left(m^{(\mu)}, p^{(\mu)}\right)$-regular, if 1 is $(m, p)$-entrance $(l c c)$,

(ii') $\left(m^{(\mu)}, p^{(\mu)}\right)$-pure exit, if 1 is $(m, p)$-entrance (lpc),

(iii') $\left(m^{(\mu)}, p^{(\mu)}\right)$-i.a. exit, if 1 is $(m, p)$-pure natural.

Proof. If $p(1)=\infty$ then $\lambda_{0}=0$. From $\mu>\lambda_{0}=0$ it follows $p^{(\mu)}(1)$ $=\Gamma(\mu)<\infty$, i.e. 1 is $\left(m^{(\mu)}, p^{(\mu)}\right)$-accessible or-i.a. exit. Let $\tilde{m}:=m^{(\mu)}, \tilde{p}:=p^{(\mu)}$. Then $-\mu=\lambda_{0}:=\sup \operatorname{supp} \tau^{(\mu)}<0$. We apply Proposition 5 to $(\tilde{m}, \tilde{p})$ and remark $\tilde{m}^{(-\mu)}=m, \tilde{p}^{(-\mu)}=p$ (see Lemma 1$)$.

If 1 is $(m, p)$-entrance with 1 cc then $p^{(\mu)}(1)<\infty$, i.e. 1 is $(m, p)$-accessible or $(m, p)$-i.a. exit. From Proposition 5 it follows that 1 is $(m, p)$-regular. Thus (i') holds. (ii') and (iii') are proved analogously.

Q.E.D.

\section{§4. Asymptotic Properties of $p(t, x, y)$}

Now we study the properties of $p(t, x, y)$ for $y \rightarrow 1$ or/and $t \rightarrow \infty$ under the assumption that 1 is accessible or entrance. If 1 is natural such properties as are proven below do not hold. For short formulation of the results we introduce the following notation: Let $h(t, x, y)$ and $h(t, x, 1)(t>0 ; x, y \in[0,1))$ be nonnegative measurable functions. We shall say that $h(t, x, y)$ converges for $y \rightarrow 1$ in space-weak (shortly: $s$-weak) sense to $h(t, x, 1)$ if

$$
\lim _{y \rightarrow 1} \int_{0}^{1} h(t, x, y) f(x) m(d x)=\int_{0}^{1} h(t, x, 1) f(x) m(d x) \quad\left(f \in C_{c}, t>0\right)
$$

where $C_{c}:=\{f \in C \mid f(x)=0$ for $x \in[a, 1)$ and some $a<1\}$.

We shall say that $h(t, x, y)$ converges for $y \rightarrow 1$ in time-weak (shortly: $t$-weak) sense to $h(t, x, 1)$ if

$$
\lim _{y \rightarrow 1} \int_{0}^{t} h(s, x, y) d s=\int_{0}^{t} h(s, x, 1) d s \quad(x \in[0,1), t>0) .
$$

If $h(t, x, y)$ converges to $h(t, x, 1)$ in $s$ - and $t$-weak sense, we write st-lim $\lim _{y \rightarrow 1} h(t, x, y)$ $=h(t, x, 1)$.

Now we can formulate the following

Theorem 2. (i): Let 1 be entrance. Then

$$
\lim _{y \rightarrow 1} \frac{p(t, x, y)}{p(y)}=0 \quad(x \in[0,1), t>0)
$$

pointwise land there exists a strictly positive and continuous function $p(t, x, 1)$ with 


$$
\text { st- } \lim _{y \rightarrow 1} p(t, x, y)=p(t, x, 1) \quad(x \in[0,1), t>0) .
$$

(ii): Let 1 be accessible. Then

$$
\lim _{y \rightarrow 1} p(t, x, y)=0 \quad(x \in[0,1), t>0)
$$

pointwise and there exists a strictly negative and continuous function $D_{p} p(t, x, 1)$ with

$$
\text { st- } \lim _{y \rightarrow 1} \frac{p(t, x, y)}{p(y)-p(1)}=D_{p} p(t, x, 1) \quad(x \in[0,1), t>0) .
$$

Proof: From Proposition 2 and the corollary after Proposition 1 the properties (19) and (21) follow directly. Let 1 be entrance. By the theorem of Riesz for every $t>0$ there exists a measure on $[0,1]$ denoted by $P_{t}(1, d y)$ such that

$$
S_{t} f(1)=\int_{0}^{1} P_{t}(1, d y) f(y) \quad(f \in C) .
$$

From $S_{t} 1=1$ it follows $P_{t}(1,[0,1])=1$. The semigroup property of $\left(S_{t}\right)$ implies

$$
S_{t} f(1)=\left(S_{h}\left(S_{t-h} f\right)\right)(1)=\int_{0}^{1} f(z)\left(\int_{0}^{1} P_{h}(1, d y) p(t-h, y, z)\right) m(d z)
$$

$(t>0,0<h<t)$. Defining $p(t, x, 1):=\int_{0}^{1} P_{h}(1, d y) p(t-h, y, x)$ we have

$$
\lim _{y \rightarrow 1} \int_{0}^{1} f(x) p(t, x, y) m(d x)=\int_{0}^{1} f(x) p(t, x, 1) m(d x) \quad(f \in C)
$$

by continuity of $S_{t} f$ on $[0,1]$ for $f \in C$. From the continuity of $p(t-h, y, x)$ in $(t, x)$ with $x \in[0,1)$ and $t>0$ it follows that $p(t, x, 1)$ is continuous there.

Now we show that $p(t, x, 1)$ is strictly positive. Suppose conversely $p\left(t_{0}, x_{0}, 1\right)$ $=0$ for some $\left(t_{0}, x_{0}\right)$ with $t_{0}>0, x_{0} \in[0,1)$. Then $P_{h}(1,[0,1))=0$ for any $h \in\left(0, t_{0}\right)$ by definition and strong positiveness of the density $p(t-h, y, x)$. Thus $P_{h}(1,\{1\})$ $=1$ and therefore $S_{h} f(1)=f(1)$ for every $h>0$. This implies $R_{\lambda} f(1)=\frac{f(1)}{\lambda}(\lambda>0)$. But if 1 is entrance we have $0 \leqq \varphi(x, \lambda) \int_{x}^{1} f \chi(., \lambda) d m \leqq \chi(x, \lambda) \int_{x}^{1} f \varphi(., \lambda) d m \underset{x \rightarrow 1}{\rightarrow}$ in virtue of the monodromy of $\varphi$ and $\chi$ and in virtue of $D_{p} \varphi(1, \lambda)=\frac{1}{\lambda} \int_{0}^{1} \varphi(., \lambda) d m$ $<\infty$ and $f \in C$. Thus $R_{\lambda} f(1)=\chi(1, \lambda) \int_{0}^{1} \varphi f d m$, which contradicts $R_{\lambda} f(1)=\frac{f(1)}{\lambda}$. 
Hence we have proved the part (i) without the $t$-weak convergence in (20). The part (ii) of the theorem (without $t$-weak convergence in (22) we shall prove using (i) and the transformation studied in Chapter 3.

Let 1 be accessible. Then $\lambda_{0}$ :=sup supp $\tau$ is an isolated eigenvalue of $\mathbb{D}_{m} \mathbb{D}_{p}$ (see theorem 1). We define a new canonical pair $\left(m^{*}, p^{*}\right)$ by $d m^{*}=$ $\varphi^{2}\left(., \lambda_{0}\right) d m$ and $d p^{*}=\varphi^{-2}\left(., \lambda_{0}\right) d p$. A simple calculation shows that

$$
p^{*}(t, x, y)=e^{-\lambda_{0} t} \frac{p(t, x, y)}{\varphi\left(x, \lambda_{0}\right) \varphi\left(y, \lambda_{0}\right)} \quad(t>0 ; x, y \in[0,1))
$$

is the transition density corresponding to $\left(m^{*}, p^{*}\right)$.

By Proposition 5 the boundary 1 is $\left(m^{*}, p^{*}\right)$-entrance. Thus from (i) it follows the existence of a strictly positive continuous function $p^{*}(t, x, 1)(t>0$, $x \in[0,1)$ ) such that

$$
\lim _{y \rightarrow 1} \int_{0}^{1} p^{*}(t, x, y) f(x) m^{*}(d x)=\int_{0}^{1} p^{*}(t, x, 1) f(x) m^{*}(d x) \quad(f \in C, x \in[0,1), t>0) .
$$

Thus we have

$$
\lim _{y \rightarrow 1} \int_{0}^{1} \frac{p(t, x, y)}{\varphi\left(y, \lambda_{0}\right)} e^{-\lambda_{0} t} f(x) \varphi\left(x, \lambda_{0}\right) m(d x)=\int_{0}^{1} p^{*}(t, x, 1) f(x) \varphi^{2}\left(x, \lambda_{0}\right) m(d x) .
$$

Remarking that $\varphi\left(., \lambda_{0}\right)$ is continuous and strictly positive on $[0,1)$ (see Theorem 1) and $-\infty<D_{p} \varphi\left(1, \lambda_{0}\right)<0$ (see Proposition 4) it follows for $g=f \cdot \varphi\left(., \lambda_{0}\right)$

$$
\lim _{y \rightarrow 1} \frac{p(t, x, y)}{p(y)-p(1)} e^{-\lambda_{0} t} g(x) m(d x)=D_{p} \varphi\left(1, \lambda_{0}\right) \int_{0}^{1} p^{*}(t, x, 1) \varphi\left(., \lambda_{0}\right) g(x) m(d x)
$$

Defining $D_{p} p(t, x, 1):=e^{\lambda_{0} t} D_{p} \varphi\left(1, \lambda_{0}\right) \varphi\left(x, \lambda_{0}\right) p^{*}(t, x, 1)$ for $x \in[0,1), t>0$ and using that every $g \in C_{c}$ has the representation $g=f \cdot \varphi\left(., \lambda_{0}\right)$ for some $f \in C_{c}$ it follows

$$
s-\lim _{y \rightarrow 1} \frac{p(t, x, y)}{p(y)-p(1)}=D_{p} p(t, x, 1)<0 \quad(t>0, x \in[0,1)) .
$$

Thus (ii) without the $t$-weak convergence in (22) is proved.

Now we will show the $t$-weak convergence in (20) and (22). To this purpose we will use a lemma which was proven for diffusion processes in [19].

Lemma 4. Let $p(1)<\infty$. Then we have

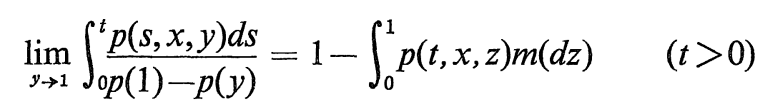

uniformly in $x \in[0, c]$ for every $c<1$. 
Proof. We assume that $y$ is a point of increasing of $m$ 。 Then (see (A6))

$$
\begin{aligned}
& \int_{0}^{t} p(s, x, y) d s=r_{0}(x, y)-\int_{t}^{\infty} p(s, x, y)=(p(1)-p(y))-\int_{0}^{\infty} p(s+t, x, y) d s \\
= & (p(1)-p(y))-\int_{0}^{1} p(t, x, z) r_{0}(z, y) m(d z) \quad(x \leqq y)
\end{aligned}
$$

and it follows

$$
\int_{0}^{t} \frac{p(s, x, y)}{p(1)-p(y)} d s=1-\int_{0}^{y} p(t, x, z) m(d z)-\int_{y}^{1} p(t, x, z) \frac{p(1)-p(z)}{p(1)-p(y)} m(d z) .
$$

Remarking that the two integrals on the right side converge for every $c<1$ uniformly in $x \leqq c$ if $y \rightarrow 1$ (the last integral to zero), (23) follows.

Let 1 be accessible. The $t$-weak convergence in (22) is proved if we can identify the limit in (23) with $\int_{0}^{t} D_{p} p(s, x, 1) d s$ i.e. if for every $f \in C_{c}$

$$
\int_{0}^{1} \int_{0}^{t} D_{p} p(s, x, 1) d s f(x) m(d x)=\int_{0}^{1}\left(\lim _{y \rightarrow 1} \int_{0}^{t} \frac{p(s, x, y)}{p(y)-p(1)} d s\right) f(x) m(d x)
$$

Using $f \in C_{c}$ the integrals on the left side of (24) can be changed. Furthermore we have

$$
\int_{0}^{1} \frac{p(s, x, y)}{p(y)-p(1)} f(x) m(d x)=\int_{0}^{1} \frac{f(x)}{\varphi\left(x, \lambda_{0}\right)} p^{*}(s, x, y) m^{*}(d x) \frac{\varphi\left(y, \lambda_{0}\right)}{p(y)-p(1)} e^{\lambda_{0} s}
$$

with $m^{*}$ and $p^{*}$ as above. This integral is majorized by $\sup _{x}\left|\frac{f(x)}{\varphi\left(x, \lambda_{0}\right)}\right|$ $\left(\left|D_{p} \varphi\left(1, \lambda_{0}\right)\right|+\varepsilon\right) e^{\lambda_{0} s}$ for $y$ sufficiently near 1 . Summarizing the above argument we have the $s$-weak convergence in (22)

$$
\begin{aligned}
& \lim _{y \rightarrow 1} \int_{0}^{1} \int_{0}^{t} \frac{p(s, x, y)}{p(y)-p(1)} d s f(x) m(d x)=\lim _{y \rightarrow 1} \int_{0}^{t} \int_{0}^{1} \frac{p(s, x, y)}{p(y)-p(1)} f(x) m(d x) d s \\
= & \int_{0}^{t} \lim _{y \rightarrow 1} \int_{0}^{1} \frac{p(s, x, y)}{p(y)-p(1)} f(x) m(d x) d s=\int_{0}^{t} \int_{0}^{1} D_{p} p(s, x, 1) f(x) m(d x) d s \\
= & \int_{0}^{1} \int_{0}^{t} D_{p} p(s, x, 1) d s f(x) m(d x) \quad\left(f \in C_{c}\right) .
\end{aligned}
$$

The left hand side of (25) can be written as $\int_{0}^{1} \lim \int_{0}^{t} \cdots$ because of the uniform convergence in Lemma 4 and $f \in C_{c}$. Thus (24) holds, i.e. the $t$-weak convergence in (22) is proved. Let 1 be entrance. We show the $t$-weak convergence in (20) by using (22) and the methods of the transformation studied in Chapter 3. 
Assume $\mu>0$. Then 1 is $\left(m^{(\mu)}, p^{(\mu)}\right)$-accessible and from (22) we have

$$
\lim _{y \rightarrow 1} \int_{0}^{t} \frac{p^{(\mu)}(s, x, y)}{p^{(\mu)}(y)-p^{(\mu)}(1)} d s=\int_{0}^{t} D_{p}^{(\mu)} p^{(\mu)}(s, x, 1) d s .
$$

By definition and a short calculation it follows that the left hand side integrals are equal to

$$
\int_{0}^{t} p(s, x, y) d s \cdot(\varphi(x, \mu) \chi(y, \mu))^{-1} .
$$

Remarking that $\chi(1, \mu)>0$ and that the limit in (26) is uniformly in $x \leqq c<1$ (see Lemma 4), we have that $\lim _{y \rightarrow 1} \int_{0}^{t} p(s, x, y) d s$ exists uniformly in $x \leqq c$ for all $c<1$ and all $t>0$.

As in the case of accessibility of 1 we can show that for every $f \in C_{c}$ the equality $\lim _{y \rightarrow 1} \int_{0}^{1}\left(\int_{0}^{t} p(s, x, y) d s\right) f(x) m(d x)=\int_{0}^{1} \int_{0}^{t} p(s, x, 1) d s f(x)$ holds. (The calculations are left to the reader.) Thus $\lim _{y \rightarrow 1} \int_{0}^{t} p(s, x, y) d s=\int_{0}^{t} p(s, x, 1) d s$ as was to be shown. Therefore the proof of the Theorem 2 is finished.

If the boundary 1 is in the limit circle case the convergence character in Theorem 2 can be improved. Moreover in this case a spectral expansion of the limit functions holds. This is the contents of the following theorem. (We remark that in $1 \mathrm{cc}$ the boundary 1 has to be regular or entrance.)

Theorem 3. Let 1 be entrance with $\int_{0}^{1} p^{2} d m<\infty$ (let 1 be regular). Then

$$
\lim _{y \rightarrow 1} p(t, x, y)=p(t, x, 1) \quad\left(\lim _{y \rightarrow 1} \frac{p(t, x, y)}{p(y)-p(1)}=D_{p} p(t, x, 1)\right)
$$

uniformly in $x \leqq 1$ and $t \geqq t_{0}$ for any $t_{0}>0$.

Moreover the following formula holds:

$$
\begin{aligned}
& p(t, x, 1)=\sum_{0}^{\infty} e^{\lambda_{k} t} \varphi\left(x, \lambda_{k}\right) \varphi\left(1, \lambda_{k}\right) \tau_{k} \\
& \left(D_{p} p(t, x, 1)=\sum_{0}^{\infty} e^{\lambda_{k} t} \varphi\left(x, \lambda_{k}\right) D_{p} \varphi\left(1, \lambda_{k}\right) \tau_{k}\right)
\end{aligned}
$$

uniformly in $x \in[0,1], t \geqq t_{0}$ for any $t_{0}>0$.

Here $\left(\lambda_{k}\right)$ denote the eigenvalues of $\boldsymbol{D}_{m} \boldsymbol{D}_{p}$ see Theorem 1$)$.

Proof. Let 1 be entrance with $\int_{0}^{1} p^{2} d m<\infty$. Then for every $\lambda>0$ we have $\varphi(., \lambda) \in L_{2}(m)$ and therefore by the monotonity of $\varphi(., \lambda)$ and $\chi(., \lambda)$ 


$$
\begin{aligned}
& r_{\lambda}^{(2)}(x, x):=\int_{0}^{1} r_{\lambda}(x, z) r_{\lambda}(z, x) m(d z)=\chi^{2}(x, \lambda) \int_{0}^{x} \varphi^{2}(y, \lambda) m(d y) \\
& +\varphi^{2}(x, \lambda) \int_{x}^{1} \chi^{2}(y, \lambda) m(d y) \leqq 2 \chi^{2}(0, \lambda)\|\varphi(., \lambda)\|_{L_{2}(m)}^{2} .
\end{aligned}
$$

Thus the function $r_{\lambda}^{(2)}(x, x)$ is bounded in $x<1$.

From the Schwartz' inequality and the symmetry of $r_{\lambda}(x, y)$ it follows $r_{\lambda}^{(2)}(x, y)^{2} \leqq r_{\lambda}^{(2)}(x, x) r_{\lambda}^{(2)}(y, y)$. Hence $r_{\lambda}^{(2)}(x, y)$ is bounded and continuous on $[0,1]^{2}$.

We know that $\varphi\left(., \lambda_{k}\right)$ is the eigenfunction of $\boldsymbol{D}_{m} \mathbb{D}_{p}$ corresponding to $\lambda_{k}$. Thus

$$
\left(R_{\lambda} \varphi\left(., \lambda_{k}\right)\right)(x)=\frac{\varphi\left(x, \lambda_{k}\right)}{\lambda-\lambda_{k}}
$$

This and $r_{\lambda}(x,.) \in L_{2}(m)$ for every $x \in I$ imply the eigenfunction expansion

$$
r_{\lambda}^{(2)}(x, .)=R_{\lambda} r_{\lambda}(x, .)=\sum_{0}^{\infty} \frac{\varphi\left(x, \lambda_{k}\right) \varphi\left(., \lambda_{k}\right)}{\left(\lambda-\lambda_{k}\right)^{2}} \tau_{k}
$$

for every $x \in I$ in $L_{2}(m)$-sense.

As in the proof of Proposition 4 it follows that $\sum_{0}^{\infty} \frac{\varphi^{2}\left(x, \lambda_{k}\right)}{\left(\lambda-\lambda_{k}\right)^{2}} \tau_{k}$ is bounded by $r_{\lambda}^{(2)}(x, x)$ and converges uniformly in $x \leqq 1$. From an analogous estimation as (14) and (15) it follows the absolute and uniform convergence of the series in (29). In particular the series

$$
p(t, x, y)=\sum_{0}^{\infty} e^{\lambda_{k} t} \varphi\left(x, \lambda_{k}\right) \varphi\left(y, \lambda_{k}\right) \tau_{k}
$$

are absolutely and uniformly convergent in $x, y \in I, t \geqq t_{0}$ for every $t_{0}>0$. We know from Theorem 1 that $\varphi\left(., \lambda_{k}\right) \in C$. Thus (27) holds.

Let 1 be regular. Then 1 is $\left(m^{\left(\lambda_{0}\right)}, p^{\left(\lambda_{0}\right)}\right)$-entrance with $1 \mathrm{cc}$. Thus we can apply the already proved part of the Theorem 3 , and after simple calculations similarly to those in the proof of Theorem 2 we obtain (28).

Q.E.D.

Now we study the properties of $p(t, x, y)$ if $t \rightarrow \infty$. To this purpose we prove the following

Lemma 5. Let 1 be entrance and $P$ the projection $f \rightarrow \int_{0}^{1} \frac{f}{m(1)} d m$ from $C$ to the subspace $N$ of constant functions. Then $\lim _{t \rightarrow \infty}\left\|S_{t}-P\right\|=0$.

Proof. In virtue of Theorem 1 and the spectral mapping theorem the 
number 1 is a simple eigenvalue of $S_{t}(t>0)$ with the eigenspace $N$ and with $\left\|S_{t}-P\right\|<1$. Therefore $S_{t}-P=S_{t}(I-P)$. This implies $\left(S_{n t}-P\right)=\left(S_{t}(I-P)\right)^{n}$ $=\left(S_{t}-P\right)^{n}$. Thus the lemma is proved.

Theorem 4. Let 1 be accessible or entrance. Then we have

$$
\lim _{t \rightarrow \infty} e^{-\lambda_{0} t} \int_{0}^{1} \frac{p(t, x, y)}{\varphi\left(x, \lambda_{0}\right) \varphi\left(y, \lambda_{0}\right)} f(y) m(d y)=\left(\int_{0}^{1} \varphi^{2}\left(z, \lambda_{0}\right) m(d z)\right)^{-1} \int_{0}^{1} f(y) m(d y)
$$

uniformly in $x \leqq 1$ and $f \in C_{c}$ with $\|f\| \leqq 1$. If 1 is in the limit circle case then moreover

$$
\lim _{t \rightarrow \infty} e^{-\lambda_{0} t} \frac{p(t, x, y)}{\varphi\left(x, \lambda_{0}\right) \varphi\left(y, \lambda_{0}\right)}=\left(\int_{0}^{1} \varphi^{2}\left(z, \lambda_{0}\right) m(d z)\right)^{-1}
$$

uniformly in $x, y \in[0,1]$.

Remark. If 1 is entrance then $\lambda_{0}=0$ and the formulas (31) and (32) simplify to

$$
\lim _{t \rightarrow \infty} \int_{0}^{1} p(t, x, y) f(y) m(d y)=(m(1))^{-1} \int_{0}^{1} f(y) m(d y)
$$

uniformly in $x \leqq 1$ and $f \in C_{c}$ with $\|f\| \leqq 1$ and if 1 is moreover in the limit circle case, then it follows

$$
\lim _{t \rightarrow \infty} p(t, x, y)=(m(1))^{-1} \text { uniformly in } x, y \in[0,1] .
$$

Proof. Suppose 1 is entrance. From Lemma 5 it follows

$$
\lim _{t \rightarrow \infty} \sup _{\|f\| \leqq 1} \sup _{x}\left|S_{t} f(x)-P f\right|=0 .
$$

Hence $\left(31^{\prime}\right)$ i.e. (31) for the entrance case is proved. If 1 is accessible, by transformation on $(m, p)$ to $\left(m^{*}, p^{*}\right)$ (see the proof of Theorem 2) and using $\left(31^{\prime}\right)$ it follows (31). The uniform and absolute convergence in (30) and $\tau_{0}^{-1}$ $=\int_{0}^{1} \varphi^{2}\left(z, \lambda_{0}\right) m(d z)$ imply (32).

Q.E.D.

As mentioned in the first chapter the theorems above are used in [13] to study parabolic functions connected with quasidiffusions. To this aim we formulate the following

Corollary. Let $f$ be a continuous function on $E \times(0, \infty)$ with compact support. If 1 is accessible or entrance then 


$$
\lim _{t \rightarrow \infty} \int_{0}^{\infty} \int_{0}^{1} \frac{p(t-s, y, x)}{e^{\lambda_{0}(t-s)} \varphi\left(y, \lambda_{0}\right) \varphi\left(x, \lambda_{0}\right)} f(x, s) m(d x) d s=\frac{\int_{0}^{\infty} \int_{0}^{1} f(x, s) m(d x) d s}{\int_{0}^{1} \varphi^{2}\left(z, \lambda_{0}\right) m(d z)}
$$

uniformly in $y \leqq 1$.

Remark. If 1 is entrance we have $\lambda_{0}=0$ and thus (33) means

$$
\lim _{t \rightarrow \infty} \int_{0}^{\infty} \int_{0}^{1} p(t-s, y, x) f(x, s) m(d x) d s=\frac{1}{m(1)} \int_{0}^{\infty} \int_{0}^{1} f(x, s) m(d x) d s .
$$

Proof. Apply (31) to the family $\{f(., s)\}$ of continuous functions on $E$ given by the $f$, use the compactness of supp $f$ and integrate with respect to $d s$.

Q.E.D.

\section{Appendix}

Here we will summarize some notions and facts from the theory of the generalized differential operator $D_{m} D_{p}$ which we have used above. They can be found in [10] (see also [2], [7], [16]) or can be proved by standard methods of ordinary differential operators. We will follow the terminology of [10] in a slight changed manner.

Let $(m, p)$ be a canonical pair and $\vartheta$ the set of all complex-valued functions $f$ on $I=[0,1)$ such that there exist an $m$-locally integrable function $g=: D_{m} D_{p} f$ and two number $a, b \in K$ with

$$
f(x)=a+b p(x)+\int_{0}^{x}(p(x)-p(s)) g(s) m(d s) \quad(x \in I) .
$$

Every $f \in \vartheta$ is linear in $p$ on intervals where $m$ is constant. Put $D_{p} f(x):=$ $b+\int_{0}^{x} D_{m} D_{p} f d m$ and $D_{p}^{-} f(0)=b(f \in \vartheta, x \in I)$. If $m(0+)=0$ the function $D_{m} D_{p} f$ is uniquely determined (modulo $m$ ) by $f \in \vartheta$ and it holds $D_{p} f(0)=D_{p}^{-} f(0)$, if $m(0+)>0$ it is uniquely determined by $[b, f]$ with $b \in K, f \in \vartheta$. In this case $[b, f]$ is called an extended function. Especially we have

$$
D_{m} D_{p} f(0)=\frac{D_{p} f(0)-D_{p}^{-} f(0)}{m(0+)} .
$$

We shall speak about functions $f \in \vartheta$ and mean extended functions if necessary. If for a function $f$ on $I$ there exists the limit $\lim _{x \rightarrow 1} f(x)$ (finite or not) it is denoted by $f(1)$. 
Let $\varphi$ and $\psi$ be the fundamental solutions of $D_{m} D_{p} g-\lambda g=0$ (see Chapter 1). For any $\lambda \in K$ and $x \in I$ we have

$$
|\varphi(x, \lambda)| \leqq \cosh (2 p(x) m(x)|\lambda|)^{1 / 2} .
$$

For every $\lambda \in K_{0}:=K \backslash(-\infty, 0]$ there exists the finite limit $\Gamma(\lambda):=\lim _{x \uparrow 1} \frac{\psi(x, \lambda)}{\varphi(x, \lambda)}$ and has a representation $\Gamma(\lambda)=\int_{-\infty}^{0} \frac{\tau(d \mu)}{\lambda-\mu}$, where $\tau$ is a uniquely determined nondecreasing function on $(-\infty, 0]$ with $\tau(x)=\frac{1}{2}(\tau(x+)+\tau(x-))(x<0)$. It is called the main spectral function of $(m, p)$. Obviously $\Gamma($.$) has a holomorphic$ extension to $K \backslash \operatorname{supp} \tau$ and $\tau$ has the property

$$
\int_{-\infty}^{0} \frac{\tau(d \mu)}{1+|\mu|}<\infty
$$

Put $\lambda_{0}$ : $=\sup \operatorname{supp} \tau$. If $\lambda$ is a point of discontinuity of $\tau$, we have $\tau(\lambda+0)$ $-\tau(\lambda-0)=\left(\int_{0}^{1} \varphi^{2}(., \lambda) d m\right)^{-1}$. By $\chi(x, \lambda)=\Gamma(\lambda) \varphi(x, \lambda)-\psi(x, \lambda)(x \in I, \lambda \in K \backslash \operatorname{supp} \tau)$ a new solution of $D_{m} D_{p} g-\lambda g=0$ is defined. We have $\chi(., \lambda) \in L_{2}(m)$ for every $\lambda \in K \backslash \operatorname{supp} \tau$. For every $\lambda>0$ the function $\varphi(., \lambda)(\chi(., \lambda))$ is positive and strictly increasing (decreasing). For the behaviour of $\varphi$ and $\chi$ near 1 see e.g. [2], p. 166. Moreover we have $\lim _{x \rightarrow 1} \varphi(x, \lambda) D_{p} \chi(x, \lambda)=-1$, and if 1 is not regular

$$
\lim _{x \rightarrow 1} \chi(x, \lambda) D_{p} \varphi(x, \lambda)=1 \text {. }
$$

This can be proved similarly as was be done in [6] for diffusions. Let $\lambda>0$. Then

$$
0 \geqq D_{p} \chi(x, \lambda)=-1+\lambda \int_{0}^{x} \chi(s, \lambda) m(d s), \text { i.e. } \int_{0}^{x} \chi(s, \lambda) m(d s) \leqq \frac{1}{\lambda} .
$$

If 1 is accessible then $\lambda_{0}<0$ (see Theorem 1) and

$$
\begin{aligned}
& 0<\varphi\left(x, \lambda_{0}\right)<1, D_{p} \varphi\left(x, \lambda_{0}\right)<0 \\
& 0<\psi\left(x, \lambda_{0}\right)<p(x) \quad(x \neq 0)
\end{aligned} \quad(x \in I)
$$

(This follows from $\varphi\left(x, \lambda_{0}\right)>0(x \in I)$ (see Theorem 1) and a comparison theorem as in the theory of ordinary differential operators, $\psi(x, \lambda)=\int_{0}^{x} \varphi^{-2}(y, \lambda) p(d y)$ $\varphi(x, \lambda)$ and

$$
\left.\psi(x, \lambda)=p(x)+\lambda \int_{0}^{x}(p(x)-p(y)) \psi(y, \lambda) m(d y)\right)
$$


From Proposition 2 it follows

$$
\int_{A} r_{\lambda}(x, y) m(d y)=\int_{A} \int_{0}^{\infty} e^{-\lambda t} p(t, x, y) d t m(d y)
$$

for every measurable subset $A$ of $I$ and every $x \in I$. If $y$ is a point of increasing of $m$ then it implies

$$
r_{\lambda}(x, y)=\int_{0}^{\infty} e^{-\lambda t} p(t, x, y) d t m(d y) .
$$

The functions $p(t, ., y), \varphi$ and $\chi$ are linear in scale $p$ on intervals where $m$ is constant. Thus (A6) holds for all $x, y \in I$ such that $x$ and $y$ are not in the same interval where $m$ is constant.

\section{References}

[1] Ahchieser, N. I.snd Glasmann, I. M.. Theorie der linearen Operatoren im Hilbertraum, Berlin, 1975.

[2] Dym, H. and McKean, H.P.. Gaussian processes, function theory and the inverse spectral problem, New York, 1976.

[3] Elliot, Joanne, Eigenfunction expansions associated with singular differential operators, Trans. Amer. Math. Soc. 78, (1955) 406-425.

[4] Feller, W., Generalized second order differential operators and their lateral conditions, Iliuois J. Math. 2(1958) , 1-18.

[5] Groh, J., Eine Klasse eindimensionaler Markovprozesse, Dissertation A, Technische Universität Dresden, 1972.

[6] Itô, K., Stochastic processes II, Moscow. 1963 (in Russian).

[7] Itô, K. and McKean, H.P., Diffusion processes and their sample path, New York, 1964.

[8] Jörgens, K., Lineare Integraloperatoren, Stuttgart, 1970.

[9] Karlin, S., McGregor, J., The differential equations of the birth- and death processes and the Stieltjes moment problems, Trans. Amer. Math. Soc. 85 (1957) 489-546.

[10] Kac, I.S. and Krein, M. G., On the spectral functions of the string, Appendix to the Russian translation of Atkinson, Discrete and continuous boundary problems, Moscow, 1968 (in Russian). Amer. Math. Soc. Trans. (2) 103 (1974) 19-102.

[11] Krein, M. G., On linear compact operators in functional spaces with two norms, Zbirnik prac institutu matematiki Akademii nauk URSR, 9 1947, 104-129 (in Russian)

[12] — Theory of selfadjoint extensions of semibounded Hermitian operators and its applications I, II Mat. Sbornik 20 (62) (1947), 431-495, and, 21 (63) (1947), 365-404 (in Russian).

[13] Küchler, U., On parabolic functions of one-dimensional quasidiffusions, Publ. RIMS, Kyoto Univ. 16 (1980), 269-287.

[14] Lai, T. L., Space-time processes, parabolic functions and one-dimensional diffusions, Trans. Amer. Math. Soc. 175 (1973), 409-438.

[15] Langer, H., Partzsch, L. ahd Schütze, D., Über verallgemeinerte gewöhnliche Differentialoperatoren mit nichtlokalen Randbedingungen and die von ihnen erzeugten Markovprozesse, Publ. RIMS, Kyoto University, 7 (1972), 659-702.

[16] Mandl, P., Analytical treatment of one-dimensional Markov processes, Berlin, 1968. 
[17] - Spectral theory of semigroups connected with diffusion processes and its applications, Čechoslovacki Matem. Zurnal, 11 (86), (1961), 558-569.

[18] Meyer, P. A., Probability and Potentials, London, 1966.

[19] Sawyer, S., A Fatou theorem for the general one-dimensional parabolic equation, Indiana Univ. Math. J., 24 (1974), 451-498.

[20] Watanabe, S., On time-inversion of one-dimensional diffusion processes, Zeitschrift Wahrscheinlichkeitstheorie verw. Gebiete, 31 (1975), 115-124.

[21] Wentzell, A. D., On the absolute continuity of the transition probabilities of onedimensional diffusion processes, (in Russian), Teorija veroj. $i$ ee primenenije, VI (1961), 439-445.

[22] Krein, M. G., On some cases of effective of the density of an inhomogeneous string by its spectral function; Doklady Akademii Nauk SSSR 83, No. 4 (1953) 617-620 (in Russian).

[23] Langer, H., Singular generalized second order differential operators with accessible or entrance boundaries, Preprint 07-10-78, Technische Universität Dresden, Sektion Mathematik. 